\title{
Article \\ A Study on a Modified Model for the Hold Time of a Clean Extinguishing Agent
}

\author{
Goonho Kim ${ }^{1}{ }^{\circledR}$, Jee-Hun Jeong ${ }^{1}$, Hyun-Jeong Bang ${ }^{2}$ and Ho-Jun Lee ${ }^{1, *}$ \\ 1 Department of Electrical Engineering, Pusan National University, Pusan 46241, Korea; \\ windthink@pusan.ac.kr (G.K.); hemn4221@pusan.ac.kr (J.-H.J.) \\ 2 Department of Safety Engineering, Incheon National University, Incheon 22021, Korea; pong9903@korea.kr \\ * Correspondence: hedo@pusan.ac.kr; Tel.: +82-51-510-2301
}

check for updates

Citation: Kim, G.; Jeong, J.-H.; Bang, H.-J.; Lee, H.-J. A Study on a

Modified Model for the Hold Time of a Clean Extinguishing Agent. Appl.

Sci. 2021, 11, 10312. https://doi.org/ 10.3390/app112110312

Academic Editor:

Antonella D'Alessandro

Received: 23 September 2021

Accepted: 29 October 2021

Published: 3 November 2021

Publisher's Note: MDPI stays neutral with regard to jurisdictional claims in published maps and institutional affiliations.

Copyright: (c) 2021 by the authors. Licensee MDPI, Basel, Switzerland. This article is an open access article distributed under the terms and conditions of the Creative Commons Attribution (CC BY) license (https:/ / creativecommons.org/licenses/by/ $4.0 /)$.
Featured Application: The approach proposed in this paper can be applied to the fire safety field using total flooding Clean Extinguishing Agents. Accurate prediction of the hold time of a CEA can achieve reliable fire control and extinguishment, as well as prevent overdesign of the CEA system.

Abstract: When estimating the theoretical hold time of a Clean Extinguishing Agent (CEA), the hold time is predicted using a leakage exponent and leakage characteristics that are measured through an Enclosure Integrity Test (EIT). In particular, the leakage exponent $n$ is conventionally applied as 0.5 , but recently, a variable exponent has been applied through measurement (approximately 0.48 to 0.85 ). When variable $n$ is applied, the hold time varies greatly depending on the size of $n$. Therefore, in this study, a modified theoretical model for the hold time of a CEA is proposed to improve the accuracy of the hold time depending on the leakage exponent $n$. The modified theoretical model applies a different analysis of the inflow and outflow volumetric flow, and the results enable a more accurate hold time prediction. The modified theoretical equation shows an improvement of up to $8.6 \%$ for outflow volumetric flow $\dot{V}_{o}$ and a maximum improvement of approximately $10.7 \%$ for hold time $t_{h}$.

Keywords: fire extinguishing system; clean extinguishing agent; retention time; hold time; enclosure integrity test

\section{Introduction}

Water is the most widely used extinguishing agent to control, suppress, and extinguish fires. However, although water is used most efficiently, it is difficult to use in places where water damage is a concern. Therefore, in these places, water mist, gaseous agents, and a hybrid system combining water mist and inert gases are used. The water mist system extinguishes a fire by spraying very fine water droplets into the fire like water fog. That is, the sprayed water mist cools the frame and surrounding gases, displacing oxygen through evaporation, and attenuating radiant heat with the fine droplets [1-3]. The hybrid system adds the extinguishing effect of the inert agent to the extinguishing effect of the existing water mist by including inert gases in the water mist [4-6]. However, water mist has disadvantages such as high installation costs and limitations in actual discharge testing. On the other hand, a clean extinguishing agent (CEA) can limit water damage, and its performance can be evaluated without an actual discharge test through an evaluation of the theoretical model. Therefore, a CEA is one of the most widely used extinguishing agents in places where water damage is a concern. A CEA is a gas suppressant that is used in fire areas. It is discharged with a concentration above the extinguishing concentration in a sealed enclosure and prevents combustion from occurring for more than a certain period of time through physical and chemical mechanisms. This time is called the hold time or retention time. Because the hold time secures the time required to extinguish a fire 
manually, the longer this time is, the more the fire damage can be minimized [7]. Therefore, it is only possible to obtain approval from relevant authorities when evidence of a hold time exceeding the standard is secured.

Evidence of hold time in an enclosure in which a CEA is installed cannot be measured by direct discharge of an applied CEA, which can be predicted using leakage characteristics obtained from an enclosure integrity test (EIT). Theoretical models for the prediction of hold times are provided in the National Fire Protection Association (NFPA) 2001 Annex C and the International Standards Organization (ISO) 14520-1 Annex E [8,9]. The theoretical models provided are divided into the continuous mixing models and the descending interface models. The continuous mixing model is a model that is applied when the air flowing in from the outside of the enclosure and the agent-air mixture inside the enclosure are completely mixed by the bias pressure and other relevant factors. In this case, bias pressure can be generated by stack effect, wind, or a mechanical HVAC system. This model is offered by both NFPA and ISO. However, the descending interface models are different between NFPA and ISO. NFPA provides the sharp model that considers only advection, and ISO provides the wide model that considers advection and diffusion at the same time.

The difference between these two models is whether or not they consider the effect of diffusion on the prediction of hold time. Therefore, compared to the sharp model, which does not consider diffusion, the wide model predicts a conservative hold time [10,11]. This difference can be more than doubled depending on the conditions. As such, theoretical models for hold time inevitably have different predicted values depending on diffusion analysis. Todd proposed the thick model combined with the sharp model and the wide model, which reduced the prediction error of the extreme hold time of these models [11,12].

The descending interface models are divided into three types based on differences in the interpretation of diffusion, but the interpretation of advection is the same [7-10,13]. In other words, the analysis of the advection for the wide model and the thick model is the same as in the sharp model. This analysis method, proposed by DiNenno and Forssell, is still applied today [14]. This analysis method predicts the outflow volumetric flow using the leakage characteristics obtained through the EIT, and the hold time is predicted using this value. That is, the interpretation of advection proposed by DiNenno and Forssell is based on the prediction of the outflow volumetric flow out of the enclosure.

The outflow volumetric flow through the leakage area of the enclosure is derived from the general orifice theory. In this case, $n$ is the leakage exponent. It is simply expressed as an exponent of $1 / 2$ in the derivation of Bernoulli's equation, but it has a value that varies from 0.5 (turbulent flow) to 1 (laminar flow), depending on the flow of the fluid [15].

The analysis of the outflow volumetric flow formed by the density and pressure difference involves the analysis of the height of the neutral plane, which is the height at which the pressure inside and outside the enclosure equalizes. The analysis of the height of the neutral plane can be derived through the conservation of mass inside the enclosure [16,17]. In other words, it is analyzed while assuming that the inflow and outflow mass flow rate are the same. However, DiNenno and Forssell derived an equation for the outflow volumetric flow while assuming that the volume flow rate is the same rather than the mass flow rate. There is no significant difference when the leakage exponent $n$ has an approximate value of 0.5 or the difference in density inside and outside the enclosure is small. However, when the leakage exponent $n$ increases or the density difference increases, the difference in the predicted hold time according to the two assumptions increases. In the study by DiNenno and Forssell, the leakage exponent $n$ was estimated to be approximately 0.5 , but they also noted that $n$ can vary from 0.5 depending on the actual flow characteristics [14]. Early theoretical models used a fixed leakage exponent $n$ of 0.5 , so this difference could not be seen. However, a variable leakage exponent $n$ has been adopted since the 2007 edition of NFPA 2001 and the 2006 edition of ISO 14520-1. Specifically, in NFPA 2001, $n$ is suggested as a value between 0.48 and 0.85 [8]. Therefore, in this study, we propose a modified theoretical model for hold time that is interpreted as the mass flow, which we attempted to verify through theory and experiment. The experiments 
were conducted using IG-01 and HFC-125. By comparing the predicted hold time with the observed hold time, the proposed theory was validated.

\section{Theoretical Background}

\subsection{Deviation of the Theoretical Equations for the Hold Time}

An existing theoretical model for the hold time of a CEA starts from the sharp model proposed by DiNenno and Forssell [14]. The sharp model was extended to the wide model proposed by Dewsbury and Whiteley and modified to the thick model proposed by Todd $[7,11,12]$. The theoretical model is derived from the mass conservation equation for the agent inside the enclosure through several assumptions. The assumptions for the hold time of a CEA are as stated below $[7,11-14,18-22]$ :

- The protected has a constant cross-section according to its height.

- The leakage areas through the protected enclosure boundaries occur only at the upper and the lower extremes.

- The inside and outside of the protected enclosure have a standard temperature and pressure.

- For all species, diffusivity is assumed to be the same.

- Thermal effects are ignored.

- The initial state of the agent in the protected room is a homogeneous mixture.

A schematic diagram of the theoretical model for hold time is shown in Figure 1.

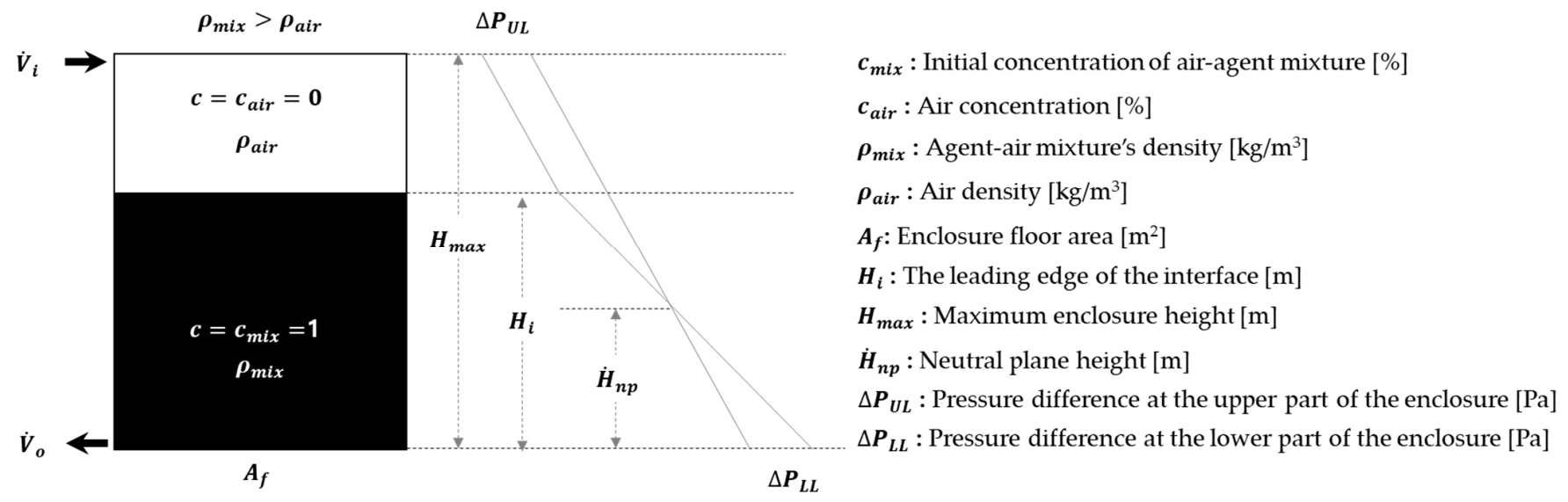

Figure 1. Schematic diagram of hydrostatic pressure profile of the sharp model.

Figure 1 shows a schematic diagram of the sharp model where the agent-air mixture inside the enclosure and the air outside do not mix. The existing theoretical equations for the hold time of the CEA were derived from the sharp model. In the sharp model, because there is no agent-air mixture flowing in, $\dot{m}_{m i x}$,in is zero. Therefore, the mass of the agent-air mixture inside the enclosure can be expressed as Equation (1).

$$
\dot{m}_{m i x}=\dot{m}_{m i x, \text { in }}-\dot{m}_{m i x, \text { out }}=-\dot{m}_{m i x, \text { out }}\left(-\dot{m}_{\text {mix }, \text { in }}=0\right)
$$

Equation (1) can be transformed into a form in which the height of the interface formed by the agent-air mixture and air decreases per unit time. This is expressed as Equation (2).

$$
\frac{\partial H_{i}}{\partial t}=-\frac{\dot{V}_{o}}{A_{f}}
$$

where

$$
\dot{m}_{m i x}=\rho_{m i x} A_{f} \dot{H}_{i} \dot{m}_{m i x, o u t}=\rho_{m i x} \dot{V}_{o}
$$


Equation (2) shows that the descending velocity of the leading edge of the interface $\dot{H}_{i}$, which is the boundary between the agent-air mixture and air, is ultimately determined by the outflow volumetric flow $\dot{V}_{o}$ discharged from the enclosure. Therefore, the time it takes for $\dot{H}_{i}$ to descend to the required protected height $H_{p}$ for which the hold time is determined is called the hold time, and $H_{p}$ is usually set as the point of maximum combustibles or $75 \%$ of the maximum height of the enclosure [11,18]. The theoretical equation for the outflow volumetric flow $\dot{V}_{o}$ is expressed in the form of a power law function as in Equation (3).

$$
\dot{V}_{o}=k_{1} \Delta P^{n}
$$

As shown in Equation (3), $\dot{V}_{o}$ can be determined from the pressure difference inside and outside the enclosure. The pressure difference inside and outside the enclosure can be seen in Equations (4) and (5).

$$
\begin{gathered}
\Delta P_{L L}=\left(\rho_{\text {mix }}-\rho_{\text {air }}\right) g \dot{H}_{n p} \\
\Delta P_{U L}=\left(\rho_{\text {mix }}-\rho_{\text {air }}\right) g\left(\dot{H}_{i}-\dot{H}_{n p}\right)
\end{gathered}
$$

where $g$ is the acceleration due to gravity.

The pressure difference inside and outside the enclosure can be predicted through the analysis of the height of the neutral plane $\dot{H}_{n p}$, and $\dot{H}_{n p}$ can be predicted through the conservation of mass within the enclosure $\left(\rho_{\text {mix }} \dot{V}_{o}=\rho_{\text {air }} \dot{V}_{i}\right)$. However, the existing theoretical model was derived by assuming that the inflow and outflow volume flows are the same $\left(\dot{V}_{o}=\dot{V}_{i}\right)$. The outflow volumetric flow $\dot{V}_{o}$ and hold time $t_{h}$ are calculated through the same volume flow as follows:

$$
\begin{gathered}
\dot{V}_{o}=A_{o} C_{d} C_{u}\left(\frac{2 \Delta P_{L L}}{\rho_{\text {mix }}}\right)^{n}=A_{o} C_{d} C_{u}\left\{\left(\frac{2}{\rho_{\text {mix }}}\right)\left[\frac{\left(\rho_{\text {mix }}-\rho_{\text {air }}\right) g \dot{H}_{i}}{1+\left(\frac{\rho_{\text {air }}}{\rho_{\text {mix }}}\right)\left(\frac{A_{o}}{A_{i}}\right)^{\frac{1}{n}}}\right]\right\}^{n} \\
t_{h}=\frac{V}{H_{\max }}\left[\frac{\left(k_{3} H_{\text {max }}+k_{4}\right)^{1-n}-\left(k_{3} H_{e}+k_{4}\right)^{1-n}}{(1-n) k_{2} F k_{3}}\right] \quad\left(\rho_{\text {mix }}>\rho_{\text {air }}\right) \\
t_{h}=\frac{V}{H_{\text {max }}}\left[\frac{\left(k_{3} H_{\text {max }}+k_{4}\right)^{1-n}-\left(k_{3}\left(H_{\text {max }}-H_{e}\right)+k_{4}\right)^{1-n}}{(1-n) k_{2}(1-F) k_{3}}\right] \quad\left(\rho_{\text {mix }}<\rho_{\text {air }}\right)
\end{gathered}
$$

where

$$
k_{2}=k_{1}\left(\frac{\rho_{\text {air }}}{2}\right)^{n}, k_{3}=\frac{2 g\left|\rho_{\text {mix }}-\rho_{\text {air }}\right|}{\rho_{\text {mix }}+\rho_{\text {air }}\left(\frac{F}{1-F}\right)^{1 / n}}, k_{4}=\frac{2\left|P_{\text {bh }}\right|}{\rho_{\text {mix }}+\rho_{\text {air }}\left(\frac{F}{1-F}\right)^{1 / n}}
$$

Through the EIT, the leakage exponent $n$, leakage constant $k_{1}$, bias pressure $P_{b h}$, and lower leakage fraction $F$ are measured or estimated, and the hold time $t_{h}$ can be predicted by calculating $k_{2}, k_{3}$, and $k_{4}$ using these values $[8,9]$. The hold time of the wide and thick model, which considers diffusion as well as advection, is predicted by modifying the equation above. The wide and thick model evaluates the effect on diffusion by changing the specified height $H_{e}$. That is, by setting the value of $H_{e}$ to be larger than in the sharp model, a shorter hold time is predicted. The specified height $H_{e}$ for each model is as follows [7-9,11,12].

$$
\begin{gathered}
H_{e, s d i}=H_{p} \\
H_{e, w d i}=H_{\text {max }}-\left(H_{\text {max }}-H_{p}\right)\left(\frac{c_{\text {mix }}}{2 c_{t h}}\right)
\end{gathered}
$$




$$
\begin{gathered}
H_{e, t d i}=H_{e, t d i} \quad\left(\omega_{\max } H_{\max } \geq H_{e, w d i}\right) \\
H_{e, t d i}=H_{p}-\omega H_{\max }\left(\frac{c_{\operatorname{mix}}}{c_{t h}}-\frac{1}{2}\right) \quad\left(\omega_{\max } H_{\max }<H_{e, w d i}\right)
\end{gathered}
$$

where

$$
\omega=\frac{\Delta H / H_{\max }}{\Delta c / c_{\operatorname{mix}}}
$$

In Equations (9)-(12) above, it can be seen that the wide and thick models considering diffusion are calculated by setting only the specified height $H_{e}$ differently in the sharp model. In other words, it can be seen that the prediction for hold time is greatly affected by the accuracy of the theoretical equation of the sharp model, which only considers advection.

\subsection{Consideration of the Theoretical Models}

In the previous section, the existing theoretical model was derived under the assumption that the inflow and outflow volumetric flow rates of the enclosure are the same. However, the interpretation of the neutral plane height $\dot{H}_{n p}$ in terms of hydrodynamics is derived in terms of mass flow $[12,23,24]$. Therefore, the theoretical model derived from the point of view of the volume flow needs to be reinterpreted from the point of view of the mass flow. The inflow and outflow mass flow through the leakage area can be expressed as:

$$
\begin{aligned}
\dot{m}_{o} & =\rho_{\text {mix }} \dot{V}_{o} \\
\dot{m}_{i} & =\rho_{\text {air }} \dot{V}_{i}
\end{aligned}
$$

If the outflow and inflow mass flows are the same $\left(\dot{m}_{0}=\dot{m}_{i}\right)$, the hydrostatic pressure difference at the top of the enclosure can be expressed as Equation (15).

$$
\Delta P_{U L}=\left(\frac{\rho_{\text {air }}}{\rho_{\text {mix }}}\right)^{\frac{1-n}{n}}\left(\frac{A_{o}}{A_{i}}\right)^{\frac{1}{n}} \Delta P_{L L}
$$

where $A_{i}$ and $A_{o}$ are the summed orifice areas of all upper and lower leakages, respectively.

The sum of the pressure difference between the top and bottom of the enclosure is the same as Equation (16), and by substituting Equation (15) into Equation (16), $\Delta P_{L L}$ can be expressed as Equation (17).

$$
\begin{aligned}
& \Delta P_{L L}+\Delta P_{U L}=\left(\rho_{\text {mix }}-\rho_{\text {air }}\right) g H_{i} \\
& \Delta P_{L L}=\left[\frac{\left(\rho_{\text {mix }}-\rho_{\text {air }}\right) g H_{i}}{1+\left(\frac{\rho_{\text {air }}}{\rho_{\text {mix }}}\right)^{\frac{1-n}{n}}\left(\frac{A_{o}}{A_{i}}\right)^{\frac{1}{n}}}\right]
\end{aligned}
$$

Therefore, the modified outflow volumetric flow $\dot{V}_{m o}$ analyzed from the point of view of the mass flow can be derived as Equation (18).

$$
\dot{V}_{\text {mo }}=A_{o} C_{d} C_{u}\left\{\left(\frac{2}{\rho_{\text {mix }}}\right)\left[\frac{\left(\rho_{\text {mix }}-\rho_{\text {air }}\right) g H_{i}}{1+\left(\frac{\rho_{\text {air }}}{\rho_{\text {mix }}}\right)^{\frac{1-n}{n}}\left(\frac{A_{o}}{A_{i}}\right)^{\frac{1}{n}}}\right]\right\}^{n}
$$

When comparing Equations (6) and (18), the difference between $\dot{V}_{o}$ and $\dot{V}_{m o}$ is the presence or absence of a power of $(1-n) / n$ in $\rho_{\text {air }} / \rho_{\text {mix }}$ of the denominator of the equation, and $\dot{V}_{m o} / \dot{V}_{o}$ can be expressed as Equation (19).

$$
\frac{\dot{V}_{m o}}{\dot{V}_{o}}=\left(\frac{\rho_{a i r}}{\rho_{m i x}}\right)^{\frac{2 n-1}{n}}
$$


In Equation (19), if the leakage exponent $n$ is 0.5 , it can be seen that $\dot{V}_{m o}$ and $\dot{V}_{o}$ are the same value. However, if $n$ changes, $\dot{V}_{m o} / \dot{V}_{o}$ is calculated as shown in Figure 2.

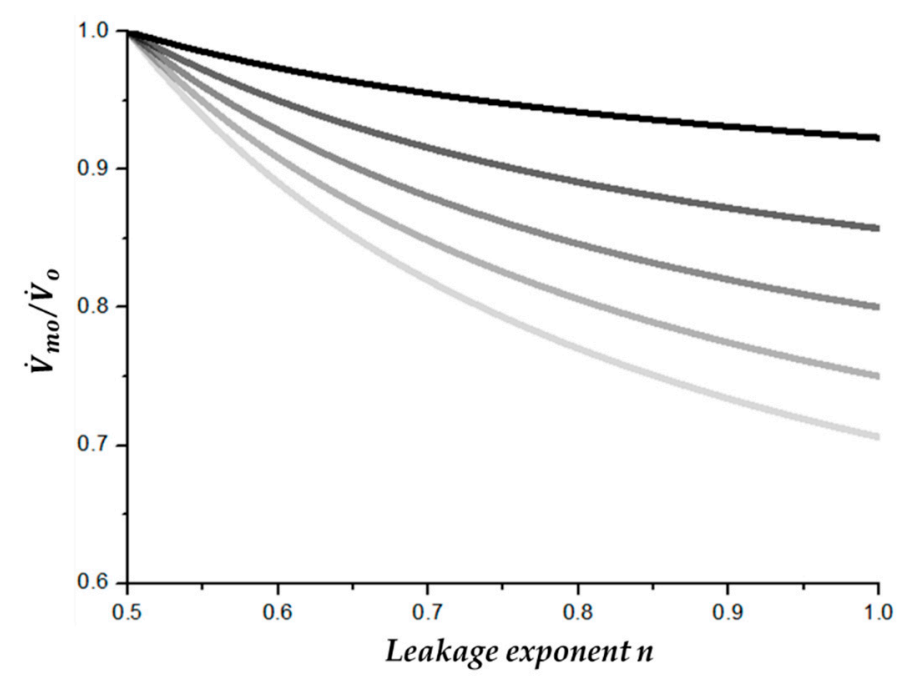

Figure 2. Schematic diagram of $\dot{V}_{m o} / \dot{V}_{o}$ change with change of leakage exponent $n$. A lighter shade indicates that the density of the agent-air mixture increases.

In Figure 2, it can be seen that the value of $\dot{V}_{m o} / \dot{V}_{o}$ decreases as the leakage exponent $n$ increases. In other words, when $n$ increases to more than $0.5, \dot{V}_{m o}$ has a value that becomes smaller than $\dot{V}_{o}$. Therefore, because the hold time is inversely proportional to outflow volumetric flow, it can be predicted that the modified hold time $t_{m h}$ will increase compared to the existing hold time $t_{h}$. The theoretical equations for $t_{m h}$ are identical to Equations (4) and (5) from the existing model. Only the values of the simplifying constants $k_{3}$ and $k_{4}$ are different, and these values can be defined as follows:

$$
\begin{aligned}
& k_{m 3}=\frac{2 g\left|\rho_{\text {mix }}-\rho_{\text {air }}\right|}{\rho_{\text {mix }}\left[1+\left(\frac{\rho_{\text {air }}}{\rho_{\text {mix }}}\right)^{\frac{1-n}{n}}\left(\frac{F}{1-F}\right)^{1 / n}\right]} \\
& k_{m 4}=\frac{2\left|P_{\text {bh }}\right|}{\rho_{\text {mix }}\left[1+\left(\frac{\rho_{\text {air }}}{\rho_{\text {mix }}}\right)^{\frac{1-n}{n}}\left(\frac{F}{1-F}\right)^{1 / n}\right]}
\end{aligned}
$$

Therefore, the difference between the existing and modified hold time will eventually show a difference according to the calculation of the simplifying constants $k_{m 3}$ and $k_{m 4}$. Also, it is expected that the difference in the predicted hold time will become larger as the leakage exponent $n$ increases and the density of agent-air mixture increases. This was validated through experimentation.

\subsection{Validation Method}

The modified outflow volumetric flow $\dot{V}_{m o}$ can be validated through two methods. First, it can be verified by comparing the theoretical hold time predicted through $\dot{V}_{m o}$ with the hold time observed in the test enclosure. This can be verified directly by measuring the concentration of the agent-air mixture observed at a specific height. The second method is to compare the predicted value and the measured value of outflow volumetric flow. However, it is difficult to measure outflow volumetric flow directly in a test enclosure. Therefore, in this study, the outflow volumetric flow was calculated using the agent concentration data observed at a specific height and compared with the existing model's $\dot{V}_{v o}$ and the modified model's $\dot{V}_{m o}$. The prediction of outflow volumetric flow according to the agent concentration can be confirmed through a theoretical model for the hold time of the CEA. 
The theoretical model for the prediction of hold time is expressed in one dimension through zone model analysis, as shown in Figure 3.

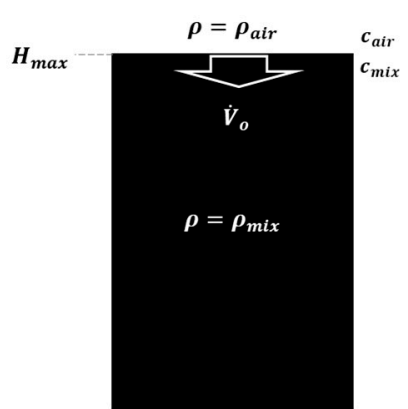

(a)

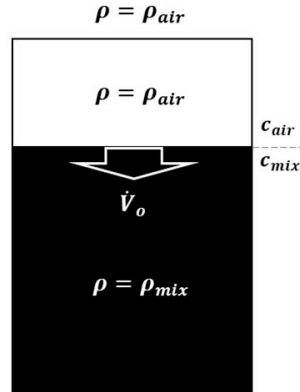

(b)

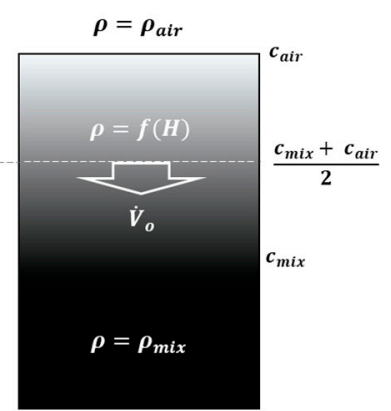

(c)

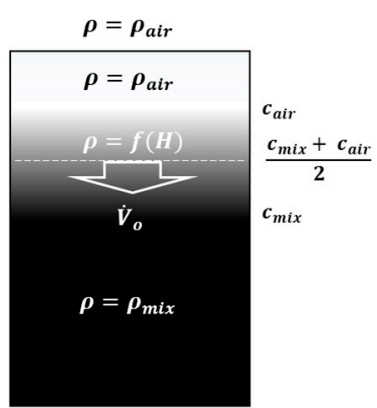

(d)

Figure 3. Schematic diagram of agent concentration distribution inside enclosure $\left(\rho_{\text {mix }}>\rho_{\text {air }}\right)$ : (a) Initial concentration distribution interpreted as a homogeneous mixture after agent discharge. Agent concentration distribution from (b) the sharp model, (c) the wide model, and (d) the thick model. (b-d) show the agent concentration distribution when $t=t_{h}$.

Figure 3a shows a schematic diagram of the initial agent concentration distribution inside the enclosure. All theoretical models for the hold time of the CEA start from the initial condition, as shown in Figure 3a. The air outside the enclosure and the agent-air mixture inside the enclosure are formed with a boundary of $H_{\max }$. The z-direction driving force is formed inside the enclosure by the outflow volumetric flow from the enclosure, and the incoming air descends downward. This is the descending interface theory.

Figure $3 b-d$ shows the concentration distribution inside the enclosure at a specific time $t=t_{h}$ for each model. The difference in the concentration distribution of each model is the difference in interpretation of diffusion. The sharp model, which does not consider species diffusion, has zero thickness of the interface between the agent-air mixture and air. Therefore, the concentration distribution inside the enclosure is only $c_{m i x}$ and $c_{a i r}$, and the hold time is interpreted optimistically. The wide model interprets species diffusion as a function of height $c=f(H)$. Here, it is assumed that species diffusion can form from the ceiling to the floor of the enclosure. Therefore, the interface thickness can be widely distributed, and the hold time is interpreted conservatively.

The thick model is a combination of the sharp model and the wide model. This model also interprets species diffusion as a function of height $c=f(H)$. However, it can be interpreted that the interface thickness does not grow beyond a certain thickness, so it is assumed that the interface thickness descends after a certain time while maintaining the same value, and the hold time has a value between that of the sharp model and that of the wide model. However, the interpretation of advection for these three models is the same.

According to the diffusion theory of fluids, if there are two fluids, as shown in Figure 3a, they diffuse up and down around the center of concentration, $\left(c_{m i x}+c_{a i r}\right) / 2[25,26]$. If there is also advection, the interface formed by diffusion around the axis of $\left(c_{\text {mix }}+c_{\text {air }}\right) / 2$ moves in the direction in which the advection is formed $[27,28]$. This is also confirmed in Figure $3 \mathrm{~b}-\mathrm{d}$. For the wide model, in Figure 3c, the descent by advection of the inflow air is expressed as a value of $\left(c_{\text {mix }}+c_{\text {air }}\right) / 2$. This is the same height as the interface of the sharp model in Figure $3 \mathrm{~b}$, which does not consider species diffusion.

This can also be verified in Equation (10). In Equation (10), if $c_{t h}=0.5 c_{m i x}$, then $H_{e, w d i}=H_{e, s d i}$ (in this equation, it is interpreted as $c_{a i r}=0$ ). That is, $c_{t h}=0.5 c_{\text {mix }}$ only expresses the descent by advection without diffusion. This is the same for the thick model in Figure $3 \mathrm{~d}$. Based on this theory, if the time and height at which the concentration inside the enclosure becomes $\left(c_{\text {mix }}+c_{\text {air }}\right) / 2$ can be measured, the outflow volumetric flow can be predicted through Equation (2). Therefore, in this study, the outflow volumetric flow was measured through this method, and the modified model was validated by comparing the 
value with the existing outflow volumetric flow $\dot{V}_{v o}$ and the modified outflow volumetric flow $\dot{V}_{m o}$.

\section{Experimental Study}

All tests conducted in this study were performed under the same environmental conditions in a self-manufactured test enclosure. A schematic diagram of the test enclosure can be seen in Figure 4.

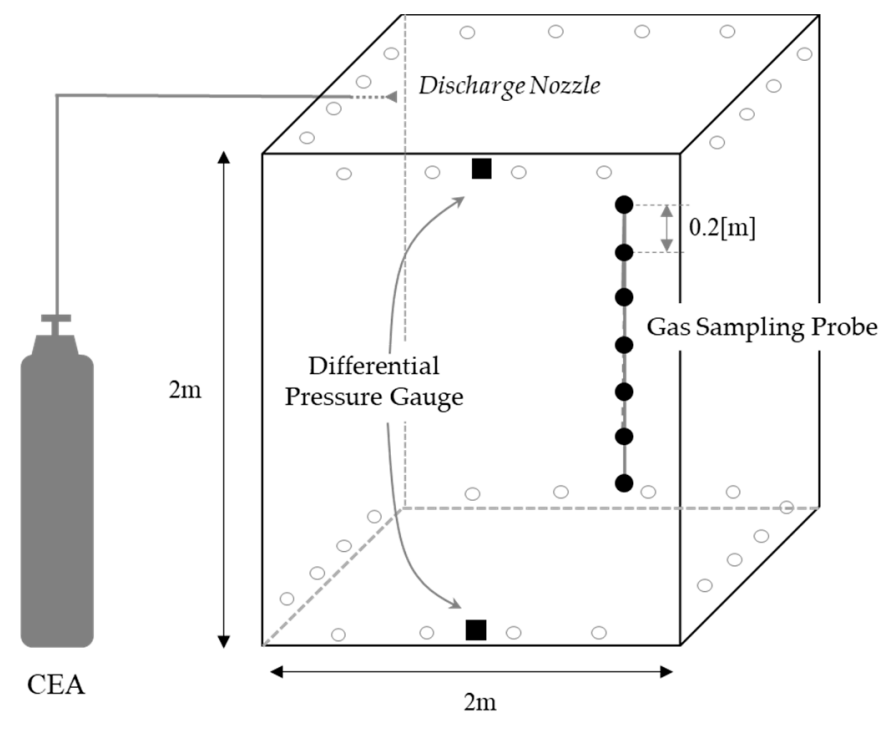

Figure 4. Schematic diagram of test enclosure used in the experiment.

The dimensions of the test enclosure were $2 \mathrm{~m} \times 2 \mathrm{~m} \times 2 \mathrm{~m}$. At the top and bottom of the test enclosure, there were 16 circular leakage holes, each with a diameter of 0.01 $\mathrm{m}$. The leakage holes were $0.05 \mathrm{~m}$ away from the ceiling and floor boundaries. The size of the outflow volumetric flow was controlled by blocking or eliminating leakage holes using vacuum plugs. The differential pressure inside and outside the test enclosure was measured using a differential pressure gauge.

The test enclosure was installed in a place that is about 30 times larger than the enclosure's volume that minimizes the change in pressure outside the enclosure due to the agent-air mixture discharged from the enclosure. It also minimizes the change in pressure inside the enclosure due to external wind $[11,12,14]$. There is no HVAC system installed in the test enclosure. Therefore, when calculating the hold time, the influence of bias pressure was not considered.

In order to apply the same assumptions of the existing theoretical model, thermal effects were not considered. The thermal effects are discussed elsewhere [10,12,16,22,29]. After the CEA was discharged into the test enclosure, all leakage areas were kept closed for at least $10 \mathrm{~min}$ to stabilize the flow of the agent-air mixture inside the enclosure and bring the temperature difference inside and outside the enclosure to within $1 \mathrm{~K}$. To establish the same initial conditions for replicates, the test enclosure was always purged with compressed air after one experiment was completed [12]. The CEA concentrations were calculated based on the oxygen concentration of the agent-air mixture. Concentrations were simultaneously measured at 7 points in $0.2 \mathrm{~m}$ intervals from $0.6 \mathrm{~m}$ to $1.8 \mathrm{~m}$ on the floor boundary. The concentration-measuring devices' linearity was secured through calibration.

The leakage exponent $n$ and leakage characteristics $k$ were measured using the fan pressurization method, and an evaluation was performed for a total of 4 conditions, as shown in Table 1. The upper and lower leakage holes were evaluated in equal numbers to set the lower leakage fraction $F$ to 0.5 . In this study, the change in lower leakage fraction $F$ was not evaluated. The agents used in the experiment were IG-01 and HFC-125, and a total 
of 5 experiments were repeated for each agent. The initial conditions of the test enclosure are shown in Table 2.

Table 1. Measured leakage exponent and leakage characteristics.

\begin{tabular}{|c|c|c|c|}
\hline $\begin{array}{l}\text { Open } \\
\text { Holes }\end{array}$ & $\begin{array}{l}\text { Leakage Exponent } n \\
\qquad(-)\end{array}$ & $\begin{array}{l}\text { Leakage Characteristics } k \\
\left(\left[/ / s / P a^{n}\right)\right.\end{array}$ & r-sq \\
\hline 4 & 0.526 & 1.275 & 0.992 \\
\hline 8 & 0.767 & 1.705 & 0.997 \\
\hline 12 & 0.609 & 2.354 & 0.999 \\
\hline 16 & 0.834 & 3.025 & 0.999 \\
\hline
\end{tabular}

Table 2. Initial conditions of test enclosure.

\begin{tabular}{ccc}
\hline & Discharge Concentration (Vol \%) & Mixture Density (kg/m $\left.\mathbf{m}^{\mathbf{3}}\right)$ \\
\hline$I G-01$ & $38 \%$ & 1.40 \\
$H F C-125$ & $8.5 \%$ & 1.63 \\
\hline
\end{tabular}

\section{Results}

The hold time of the CEA was predicted through a theoretical equation that was interpreted as a function of the height of the enclosure. The theoretical model was based on the leakage characteristics measured through an EIT and the outflow volumetric flow calculated through the leakage exponent, to predict the hold time according to the height. In a previous section, the leakage exponent and leakage characteristics were evaluated according to the number of leakage holes (Table 1). These values can be used to predict the existing outflow volumetric flow $\dot{V}_{v o}$ and the modified outflow volumetric flow $\dot{V}_{m o}$. The outflow volumetric flow at different enclosure heights predicted by Equations (6) and (18) is shown in Figure 5.

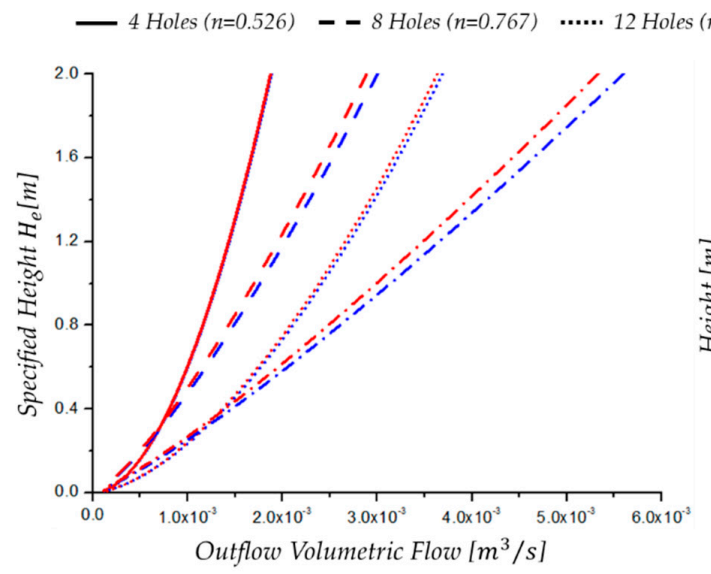

(a)

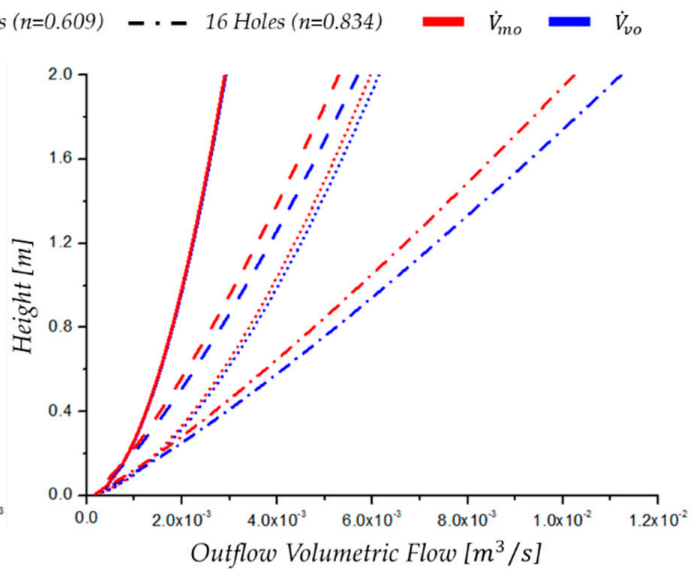

(b)

Figure 5. Outflow volumetric flow at different enclosure heights according to the number of leakage holes predicted by the existing and proposed theoretical equations: (a) IG-01; (b) HFC-125.

Different values of leakage exponent $n$ were evaluated according to the number of leakage holes. The leakage exponent $n$ was at its largest when there were 16 leakage holes and at its smallest when there were 4 leakage holes. Therefore, the outflow volumetric flows $\dot{V}_{m o}$ and $\dot{V}_{v o}$ calculated by Equations (6) and (18) can be expressed as in Figure 5.

The predicted outflow volumetric flow becomes smaller as the specified height $H_{e}$ decreases. This is a result of the neutral plane $\dot{H}_{n p}$ decreasing as time passes and the 
pressure difference $\Delta P_{L L}$ inside and outside the enclosure becomes smaller. This predicts the same result for both $\dot{V}_{v o}$ and $\dot{V}_{m o}$.

The difference between $\dot{V}_{v o}$ and $\dot{V}_{m o}$ increases as leakage exponent $n$ increases. When the number of leakage holes is 4 , the difference between $\dot{V}_{v o}$ and $\dot{V}_{m o}$ is the smallest because $n$ is the smallest as 0.526 . When the number of leakage holes is $16, n$ is the largest value (0.834), and at this time, the difference between $\dot{V}_{v o}$ and $\dot{V}_{m o}$ is also the largest. This difference is more pronounced for HFC-125 than IG-01. This happens because the agent-air mixture's density $\rho_{\text {mix }}$ of HFC- 125 is larger than that of IG-01. Therefore, it is decided that this difference will be larger in the HFC series, which has a larger $\rho_{\text {mix }}$ than the $I G$ series.

As mentioned in the previous section, it is difficult to directly experimentally measure the outflow volumetric flow of the test enclosure. Therefore, outflow volumetric flow was estimated by measuring the height and time at which the concentration of the agent-air mixture inside the enclosure became $\left(c_{m i x}+c_{a i r}\right) / 2$. The concentration distribution inside the enclosure according to the enclosure height is shown in Figure 6.

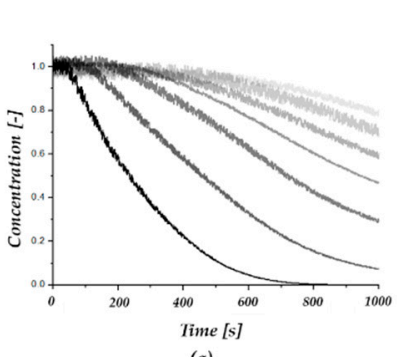

(a)

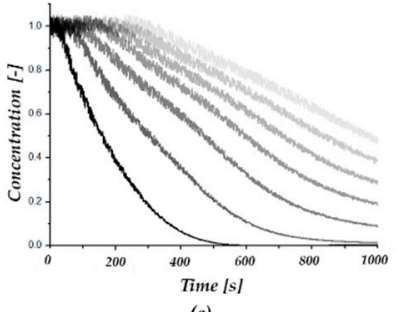

(e)

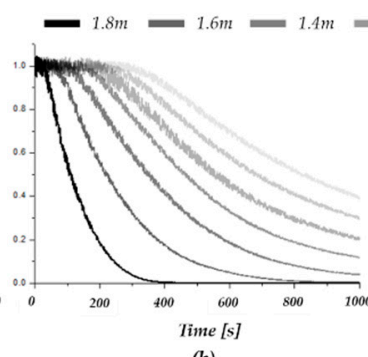

(b)

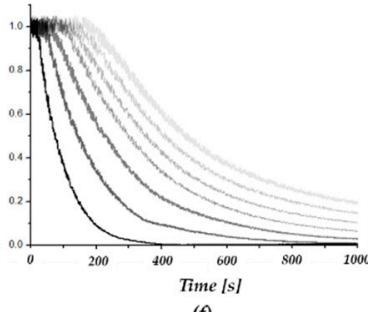

(f)

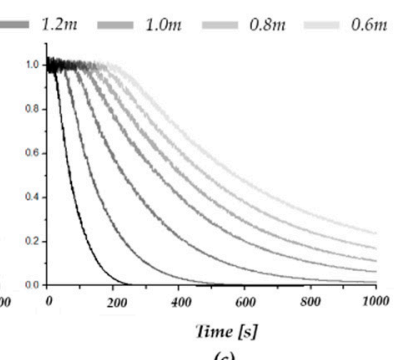

(c)

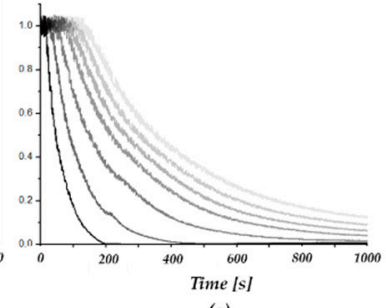

(g)

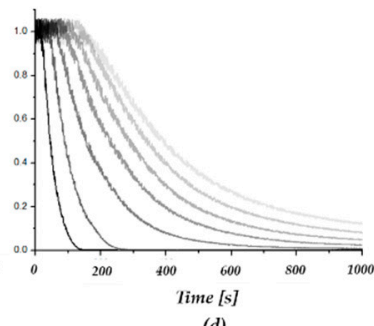

(d)

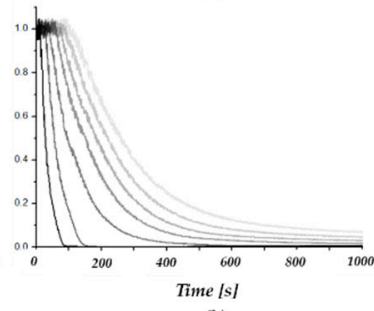

Figure 6. Concentration change according to time and enclosure height for each condition. Lighter shade indicates a decrease in the height, at which point the concentration was measured $\left(c_{\text {mix }}=1, c_{\text {air }}=0\right)$ : (a) IG-01, 4 holes; (b) IG-01, 8 holes; (c) IG-01, 12 holes; (d) IG-01, 16 holes; (e) HFC-125, 4 holes; (f) HFC-125, 8 holes; (g) HFC-125, 12 holes; and (h) HFC-125, 16 holes.

Figure 6 shows the concentration data for each height measured through the experiment. At this time, the concentration data were values converted based on the oxygen concentration measured inside the enclosure. Figure $6 \mathrm{a}-\mathrm{d}$ shows the concentration value of IG-01, and Figure 6e-h shows the concentration value of HFC-125. From these values, it can be observed that the higher the measured height is, the faster the concentration decreases. This confirms that air is coming in from the upper leakage area and is descending to the bottom.

As the number of leakage holes increases, the decrease in concentration becomes faster, and it can be seen that the decrease in concentration of HFC- 125 with a large density of agent-air mixture is faster. This result is consistent with existing studies. The predicted outflow volumetric flow at different heights based on these experimental results can be seen in Figure 7. This figure shows the outflow volumetric flow value for each height predicted based on the concentration data for each height measured in Figure 6. These values show the same trend as the theoretical outflow volumetric flow shown in Figure 5. The outflow volumetric flow increases as the number of leakage holes increases and decreases gradually as the neutral plane $\dot{H}_{n p}$ decreases. HFC-125, with a high density of agent-air mixture, has a larger outflow volumetric flow value than $I G-01$. 


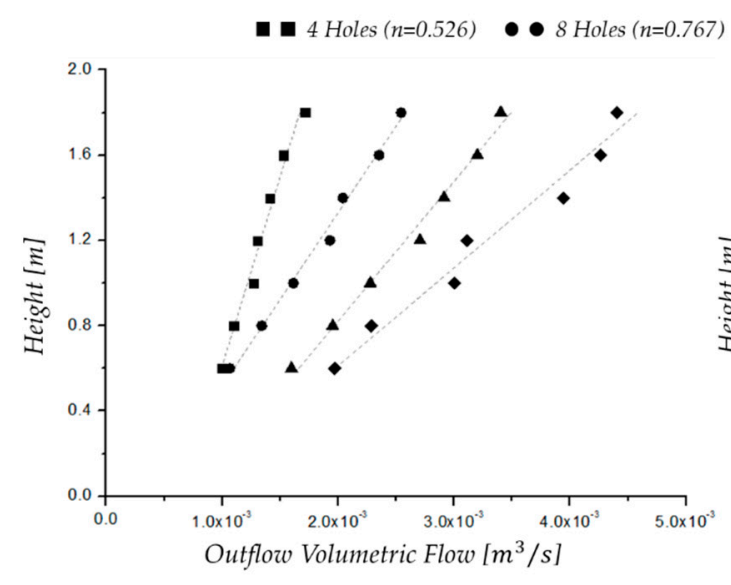

(a)

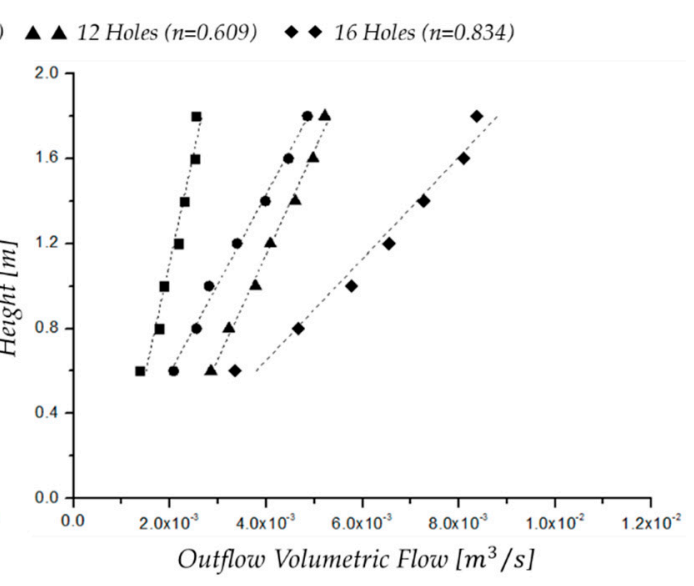

(b)

Figure 7. Outflow volumetric flow at different enclosure heights according to the number of leakage holes predicted by the existing and proposed theoretical equations: (a) IG-01; (b) HFC-125.

Figure 8 shows a comparison between the theoretical hold time predicted by $\dot{V}_{v o}$ and $\dot{V}_{m o}$ and the hold time observed in the enclosure. The theoretical hold times $t_{v h}$ and $t_{m h}$ predicted by $\dot{V}_{v o}$ and $\dot{V}_{m o}$ in Figure 8 have the same trends as the results of the outflow volumetric flow in Figure 5. The smaller the leakage exponent $n$ is, the smaller the difference is between the existing $t_{v h}$ and the modified $t_{m h}$. The larger the leakage exponent $n$ is, the larger the difference is between $t_{v h}$ and $t_{m h}$. Also, this result is more pronounced in the HFC series with large $\rho_{\text {mix }}$. The hold time observed in the test enclosure was larger than the theoretically predicted hold time values. These results were measured equally for both IG and HFC series, and the same was applied to the change in the number of leakage holes.

The validation of the proposed theory is summarized in Figures 9 and 10 and Table 3. Figures 9 and 10 show a comparison of experimentally observed and theoretically predicted results in a test enclosure. These figures demonstrate how well the theoretically predicted values can represent the experimentally observed values. The fact that the observed values are close to the dotted line means that the theoretically predicted values are highly accurate. A quantitative comparison of the observed and predicted values is shown in Table 3.

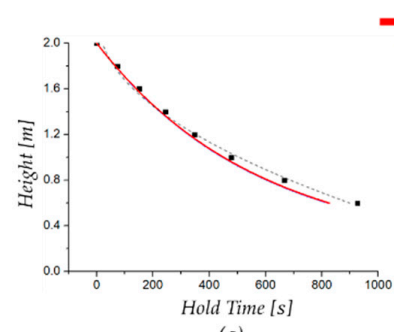

(a)

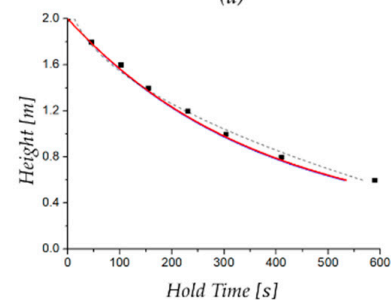

(e)

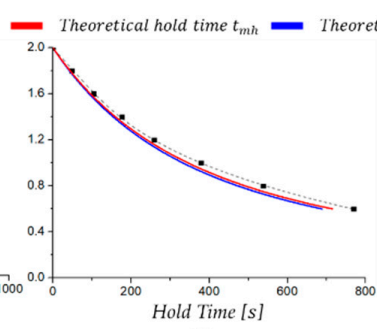

(b)

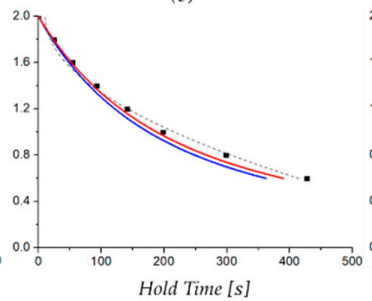

$$
\text { (f) }
$$

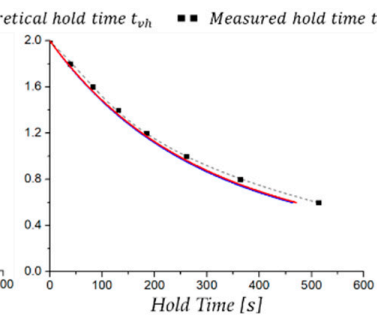

(c)

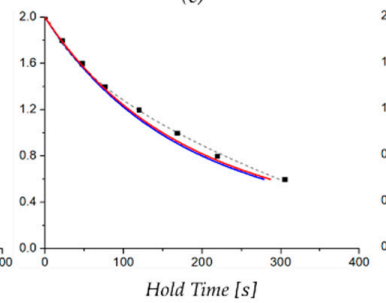

$(g)$

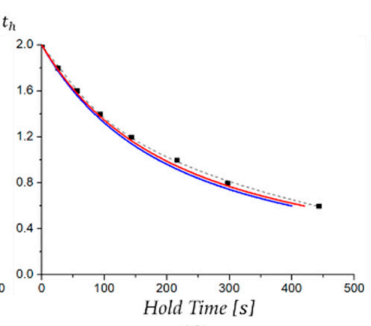

(d)

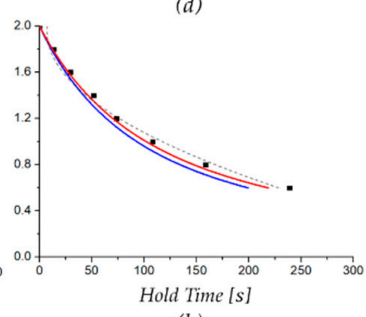

(h)

Figure 8. Change of hold time according to enclosure height for each condition. The theoretical hold time was predicted based on the wide model. The threshold concentration $c_{t h}$ at which the hold time was determined was set to $85 \%$ of the initial concentration $c_{\text {mix }}$. $\left(c_{t h}=0.85 c_{\text {mix }}\right.$ ): (a) IG-01, 4 holes; (b) IG-01, 8 holes; (c) IG-01, 12 holes; (d) IG-01, 16 holes; (e) HFC-125, 4 holes; (f) HFC-125, 8 holes; (g) HFC-125, 12 holes; and (h) HFC-125, 16 holes. 

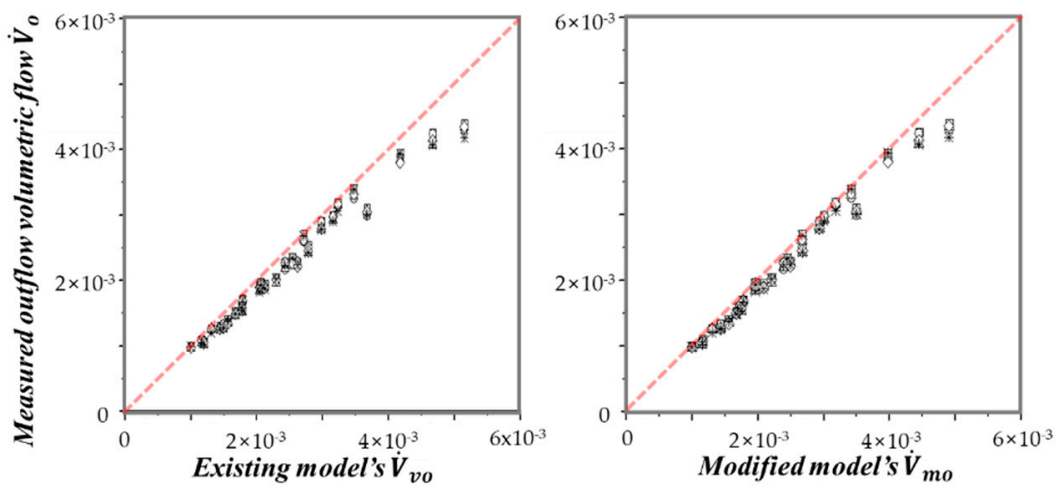

(a)

(b)

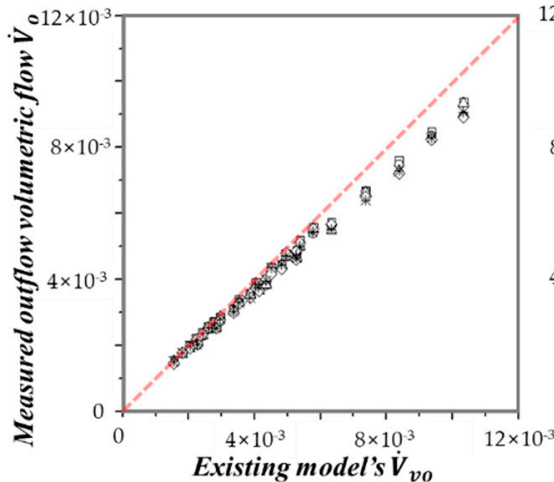

(c)

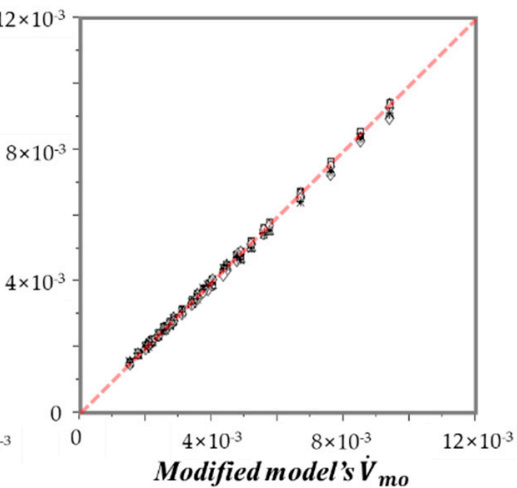

(d)

Figure 9. Comparison of theoretical and observed outflow volumetric flow $\left(c_{t h}=0.5 c_{\text {mix }}\right)$ : (a) IG-01, $\dot{V}_{v o}$ vs. $\dot{V}_{o} ;$ (b) IG-01, $\dot{V}_{m o}$ vs. $\dot{V}_{o} ;$ (c) HFC-125, $\dot{V}_{v o}$ vs. $\dot{V}_{o}$; and (d) HFC-125, $\dot{V}_{m o}$ vs. $\dot{V}_{o}$.

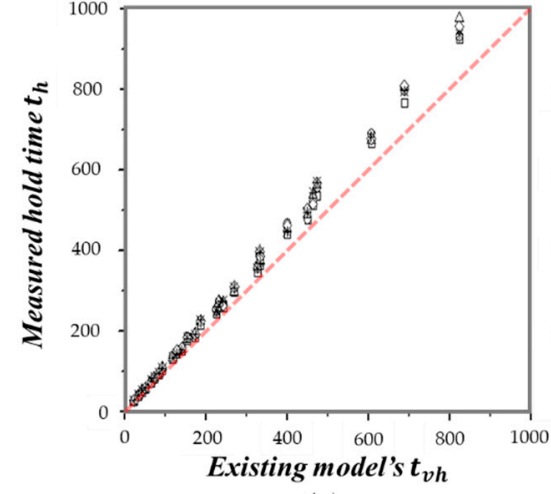

(a)

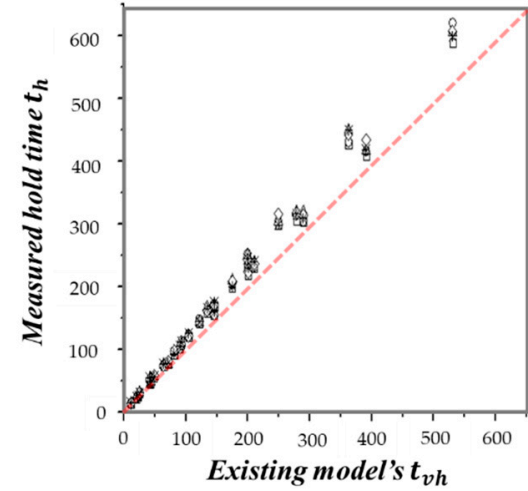

(c)

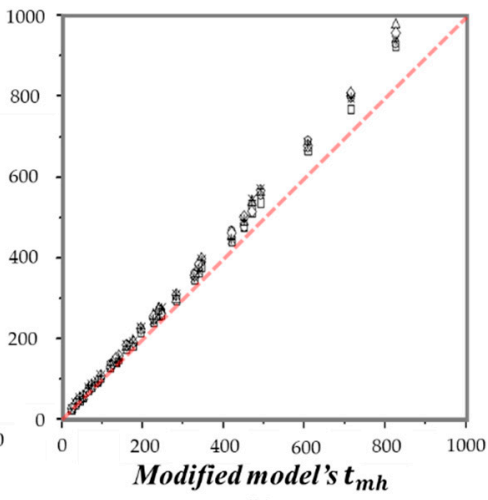

(b)

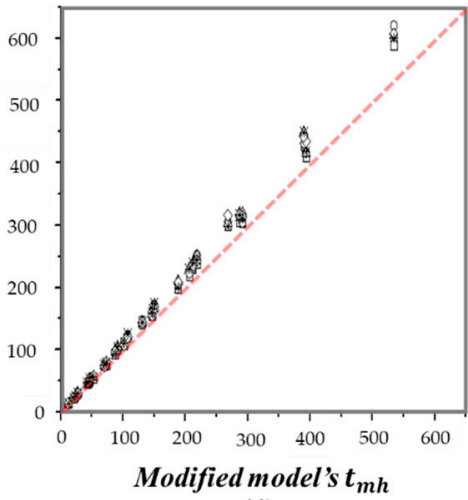

(d)

Figure 10. Comparison of theoretical and observed hold time $\left(c_{t h}=0.85 c_{m i x}\right):(a) I G-01, t_{v h}$ vs. $t_{h}$; (b) IG-01, $t_{m h}$ vs. $t_{h}$; (c) HFC- $125, t_{v h}$ vs. $t_{h}$; and (d) HFC-125, $t_{m h}$ vs. $t_{h}$. 
Table 3. Summary of theoretically predicted and experimentally observed results.

\begin{tabular}{|c|c|c|c|c|c|c|c|}
\hline & $\begin{array}{l}\text { Open } \\
\text { Holes }\end{array}$ & $\begin{array}{c}\text { Leakage } \\
\text { Exponent }\end{array}$ & $\begin{array}{c}\dot{V}_{o} / \dot{V}_{v o} \\
\text { (Mean/StDev) }\end{array}$ & $\underset{\mathbf{( \% )}}{\triangle}$ & $\begin{array}{c}t_{h} / t_{v h} \\
\text { (Mean/StDev) }\end{array}$ & $\begin{array}{c}t_{h} / t_{m h} \\
\text { (Mean/StDev) }\end{array}$ & $\underset{\mathbf{( \% )}}{\triangle}$ \\
\hline & 4 & 0.526 & $0.924 / 0.041$ & $0.3 \%$ & $1.113 / 0.031$ & $1.109 / 0.031$ & $-0.4 \%$ \\
\hline IG & 8 & 0.767 & $0.890 / 0.024$ & $3.5 \%$ & $1.172 / 0.028$ & $1.127 / 0.027$ & $-4.5 \%$ \\
\hline \multirow{3}{*}{01} & 12 & 0.609 & $0.937 / 0.039$ & $1.5 \%$ & $1.130 / 0.033$ & $1.112 / 0.032$ & $-1.8 \%$ \\
\hline & 16 & 0.834 & $0.891 / 0.047$ & $4.5 \%$ & $1.143 / 0.034$ & $1.089 / 0.033$ & $-5.5 \%$ \\
\hline & 4 & 0.526 & $0.968 / 0.017$ & $0.7 \%$ & $1.10 / 0.035$ & $1.094 / 0.035$ & $-0.7 \%$ \\
\hline HFC & 8 & 0.767 & $0.905 / 0.017$ & $6.9 \%$ & $1.18 / 0.034$ & $1.098 / 0.032$ & $-8.4 \%$ \\
\hline \multirow[t]{2}{*}{125} & 12 & 0.609 & $0.949 / 0.015$ & $2.8 \%$ & $1.13 / 0.035$ & $1.097 / 0.034$ & $-3.2 \%$ \\
\hline & 16 & 0.834 & $0.888 / 0.015$ & $8.6 \%$ & $1.21 / 0.036$ & $1.105 / 0.033$ & $-10.7 \%$ \\
\hline
\end{tabular}

In Figure 9, it can be seen that the outflow volumetric flow measured in the test enclosure is located below the dotted line as a whole. This means that the measurements are lower than the theoretically predicted values. These results become more pronounced as the number of leakage holes increases. It can be seen that the modified outflow volumetric flow $\dot{V}_{m o}$ expresses the measured outflow volumetric flow $\dot{V}_{o}$ better than the existing outflow volumetric flow $\dot{V}_{v o}$. This can be confirmed quantitatively in Table 3. In Table 3, it is shown that the value of $\dot{V}_{o} / \dot{V}_{m o}$ is closer to 1 compared to $\dot{V}_{o} / \dot{V}_{v o}$. The closer to 1 it is, the better it represents the measured outflow volumetric flow $\dot{V}_{0}$.

The same results are shown for both IG-01 and HFC-125. In particular, the larger the leakage exponent $n$ is, the better $\dot{V}_{m o}$ can express $\dot{V}_{o}$ than $\dot{V}_{v o}$. For $n=0.526$, the difference between $\dot{V}_{v o}$ and $\dot{V}_{m o}$ is not significant for both IG-01 and HFC-125. However, when $n=0.834$, IG-01 shows an improvement of approximately $4.5 \%$, and HFC- 125 shows an improvement of approximately $8.6 \%$. In other words, the larger the agent-air mixture's density $\rho_{m i x}$ is, the greater the improvement effect of $\dot{V}_{m o}$ is.

In Figure 10, it can be seen that the hold time observed in the test enclosure is located above the dotted line. This means that the observed hold time is longer than the predicted hold time because the hold time is inversely proportional to the outflow volumetric flow. Therefore, the results for hold time are similar to those for outflow volumetric flow.

The modified hold time $t_{m h}$ represents the observed hold time $t_{h}$ better than the previous hold time $t_{v h}$. Also, the larger the leakage exponent $n$ is and the larger $\rho_{m i x}$ is, the greater the improvement effect is by $t_{m h}$. For $n=0.526$, the difference between $t_{v h}$ and $t_{m h}$ is not significant $(-0.4 \%$ and $-0.7 \%$ for IG-01 and HFC-125, respectively). However, when $n=0.834$, IG-01 shows improvement of approximately $-5.5 \%$, and HFC-125 shows improvement of approximately $-10.7 \%$. The comparison of these quantitative results shows that the modified theoretical equation more accurately reflects the volume outflow and hold time than the previous model's theoretical equation. In particular, the larger the leakage exponent $n$ is and the larger the density of the agent-air mixture is, the larger the effect becomes.

\section{Conclusions}

In this study, a theoretical equation for predicting the hold time of the CEA was proposed by modifying it from the viewpoint of advection. Conventionally, when the leakage exponent $n$ is applied as 0.5 , there is no difference in the hold time predicted by the existing and modified theoretical models. However, if variable $n$ is applied, the difference in hold time predicted by the existing and modified theoretical equations increases as $n$ increases. This shows a bigger difference as $\rho_{\text {mix }}$ becomes bigger. Validation of the modified theoretical equation was done through direct comparison with the outflow volumetric flow $\dot{V}_{o}$ and hold time $t_{h}$ observed in the test enclosure.

The outflow volumetric flow $\dot{V}_{o}$ observed in the test enclosure showed more similar results to the outflow volumetric flow $\dot{V}_{m o}$ predicted by the modified equation than the 
outflow volumetric flow $\dot{V}_{v o}$ predicted by the existing theoretical equation. For the case of leakage exponent $n=0.834$, compared to the observed $\dot{V}_{o}$, the existing model's $\dot{V}_{v o}$ showed an error of approximately $11 \%$ for both IG-01 and HFC-125. However, the modified model's $\dot{V}_{m o}$ showed an error of approximately $6 \%$ for IG-01 and approximately $3 \%$ for HFC-125. This result shows an improvement effect of approximately $4.5 \%$ for IG-01 and approximately $8.6 \%$ for HFC- 125 .

The hold time also showed similar results to the outflow volumetric flow. For the case of leakage exponent $n=0.834$, compared to the observed $t_{h}$, the existing model's $t_{v h}$ showed approximately $11.4 \%$ and $21 \%$ errors for IG-01 and HFC-125, respectively. However, the modified model's $t_{m h}$ showed an error of approximately $8.9 \%$ for $I G-01$ and approximately $10.5 \%$ for $H F C-125$. This shows an improvement effect of approximately $5.5 \%$ for IG-01 and approximately $10.7 \%$ for HFC- 125 .

These results show that differences in hold time can occur depending on the interpretation of advection. It was also verified that the modified theoretical equation can predict the hold time more accurately than the existing theoretical equation. Therefore, it was decided that the modified theoretical equation could be more useful in fire-safety engineering.

Author Contributions: Conceptualization, G.K.; data curation, G.K. and H.-J.B.; formal analysis, G.K.; J.-H.J.; H.-J.B. and H.-J.L.; investigation, G.K. and J.-H.J.; project administration, H.-J.L.; writingoriginal draft preparation, G.K.; writing-review and editing, H.-J.L. All authors have read and agreed to the published version of the manuscript.

Funding: This work was supported by a 2-Year Research Grant of Pusan National University.

Institutional Review Board Statement: Not applicable.

Informed Consent Statement: Not applicable.

Data Availability Statement: The data presented in this study are available in the article.

Conflicts of Interest: The authors declare no conflict of interest.

\section{References}

1. Mawhinney, J.R. Fire protection water mist suppression systems. In Fire Protection Handbook, 18th ed.; NFPA: Quincy, MA, USA, 1997.

2. Liu, Z.; Kim, A.K. A Review of water mist fire suppression systems-Fundamental studies. J. Fire Prot. Eng. 2000, 10, 32-50. [CrossRef]

3. National Fire Portection Association. NFPA 750 Standard on Water Mist Fire Protection Systems; National Fire Protection Association: Quincy, MA, USA, 2010.

4. National Fire Protection Association. NFPA 770 Standard on Hybrid (Water and Inert Gas) Fire Extinguishing Systems; National Fire Protection Association: Quincy, MA, USA, 2021.

5. Gałaj, J.; Drzymała, T.; Wolny, P. Analysis of the impact of selected parameters of the hybrid extinguishing system on the fire environment in a closed room. Sustainability 2019, 11, 6867. [CrossRef]

6. Gałaj, J.; Tuśnio, N.; Wolny, P.; Drzymała, T. Analysis of the impact of water flow rate on the temperature variability in a closed room during the extinguishing of A-Group fire using a hybrid water mist suppression system. Sustainability 2020, 12, 8700. [CrossRef]

7. Dewsbury, J.; Whiteley, R.A. Extensions to standard hold time calculations. Fire Technol. J. 2000, 36, 266-278. [CrossRef]

8. National Fire Protection Association. NFPA 2001 Standard on Clean Agent Fire Extinguishing Systems; Annex C; National Fire Protection Association: Quincy, MA, USA, 2012.

9. ISO 14520-1 Standard on Clean Agent Fire Extinguishing Systems_Physical Properties and System Design—Part 1: General Requirements; ISO: Geneva, Switzerland, 2015.

10. Kim, G.; Cha, J.-H.; Lee, H.-J. Numerical study on the effect of temperature in the retention time of an inert agent. Fire Saf. J. 2020, 115, 103004. [CrossRef]

11. Hetrick, T.M.; Rangwala, A.S. A modified hold time model for total flooding fire suppression. Fire Saf. J. 2010, 45, 12-20. [CrossRef]

12. Hetrick, T.M. Development and Validation of a Modified Clean Agent Draining Model for Total Flooding Fire Suppression Systems; Worcester Polytechnic Institute: Worcester, MA, USA, 2008; Available online: https:/ / core.ac.uk/reader/213000180 (accessed on 23 September 2021). 
13. Mowrer, F. Analysis of vapor density effects on hold times for total flooding clean extinguishing agents. In Proceedings of the Halon Options Technical Working Conference, 16th Proceedings, HOTWC 2006, Albuquerque, NM, USA, 16-18 May 2006; pp. 1-12. Available online: https://www.nist.gov/system/files/documents/el/fire_research/R0601297.pdf (accessed on 23 September 2021).

14. DiNenno, P.J.; Forssell, E.W. Evaluation of the Door Fan Pressurization Leakage Test Method Applied to Halon 1301 Total Flooding Systems. J. Fire Prot. Eng. 1989, 1, 131-140. [CrossRef]

15. Klote, J.H. A General Routine for Analysis of Stack Effect; NISTNR 4588; National Institute of Standards and Technology: Gaithersburg, MD, USA, 1991. Available online: https://nvlpubs.nist.gov/nistpubs/Legacy/IR/nistir4588.pdf (accessed on 23 September 2021).

16. Yeoh, G.H.; Yuen, K.K. Computational Fluid Dynamics in Fire Engineering; Elsevier Inc.: Amsterdam, The Netherlands, 2009.

17. Karlsson, B.; Quintiere, J. Enclosure Fire Dynamics; CRC Press: Boca Raton, FL, USA, 1999.

18. Drewsbury, J.; Whiteley, R.A. Review of fan integrity testing and hold time standards. Fire Technol. 2000, 36, 249-265. [CrossRef]

19. Saum, D.; Saum, A.; Messing, M.; Hupman, J. Pressurization air leakage testing for Halon 1301 enclosures. In: Substitutes and Alternatives to Chlorofluorocarbons and Halon; Washington, DC, USA, 1988.

20. Fick, A. Ueber diffusion. Ann. der Phys. 1855, 170, 59-86. [CrossRef]

21. Drysdale, D. An Introduction to Fire Dynamics, 3rd ed.; John Wiley \& Sons, Ltd.: Hoboken, NJ, USA, 2011.

22. Boroń, S.; Wegrzyński, W.; Kubica, P.; Czarnecki, L. Numerical modelling of the fire extinguishing gas retention in small compartments. Appl. Sci. 2019, 9, 663. [CrossRef]

23. Simcox, S.; Wilkes, N.; Jones, I. Computer simulation of the flows of hot gases from the fire at King's Cross Underground station. Fire Saf. J. 1992, 18, 49-73. [CrossRef]

24. He, Y.; Beck, V. Estimation of neutral plane position in high rise buildings. J. Fire Sci. 1996, 14, 235-248. [CrossRef]

25. Feynman, R. The Brownian movement. In The Feynman Lectures of Physics; Pearson Education: London, UK, 1963; Volume 1, pp. 1-41.

26. Ogata, A. Theory of Dispersion in Granular Media; No. 411-1; US Geological Survey Professional Papers: Washington, DC, USA, 1970; p. 34. Available online: https:/ / pubs.usgs.gov/pp/0411i/report.pdf (accessed on 23 September 2021).

27. Bird, R.B.; Stewart, W.E.; Lightfoot, E.N. Transport Phenomena, 2nd ed.; John Wiley \& Sons, Ltd.: Hoboken, NJ, USA, 2006.

28. Crank, J. The Mathematics of Diffusion; Oxford University Press: Oxford, UK, 1980.

29. Kim, G.; Cha, J.-H.; Jeong, J.-H.; Lee, H.-J. A study on the effect of $\mathrm{O}_{2}$ diffusion on the retention time of inert agents. Appl. Sci. 2020, 10, 6694. [CrossRef] 